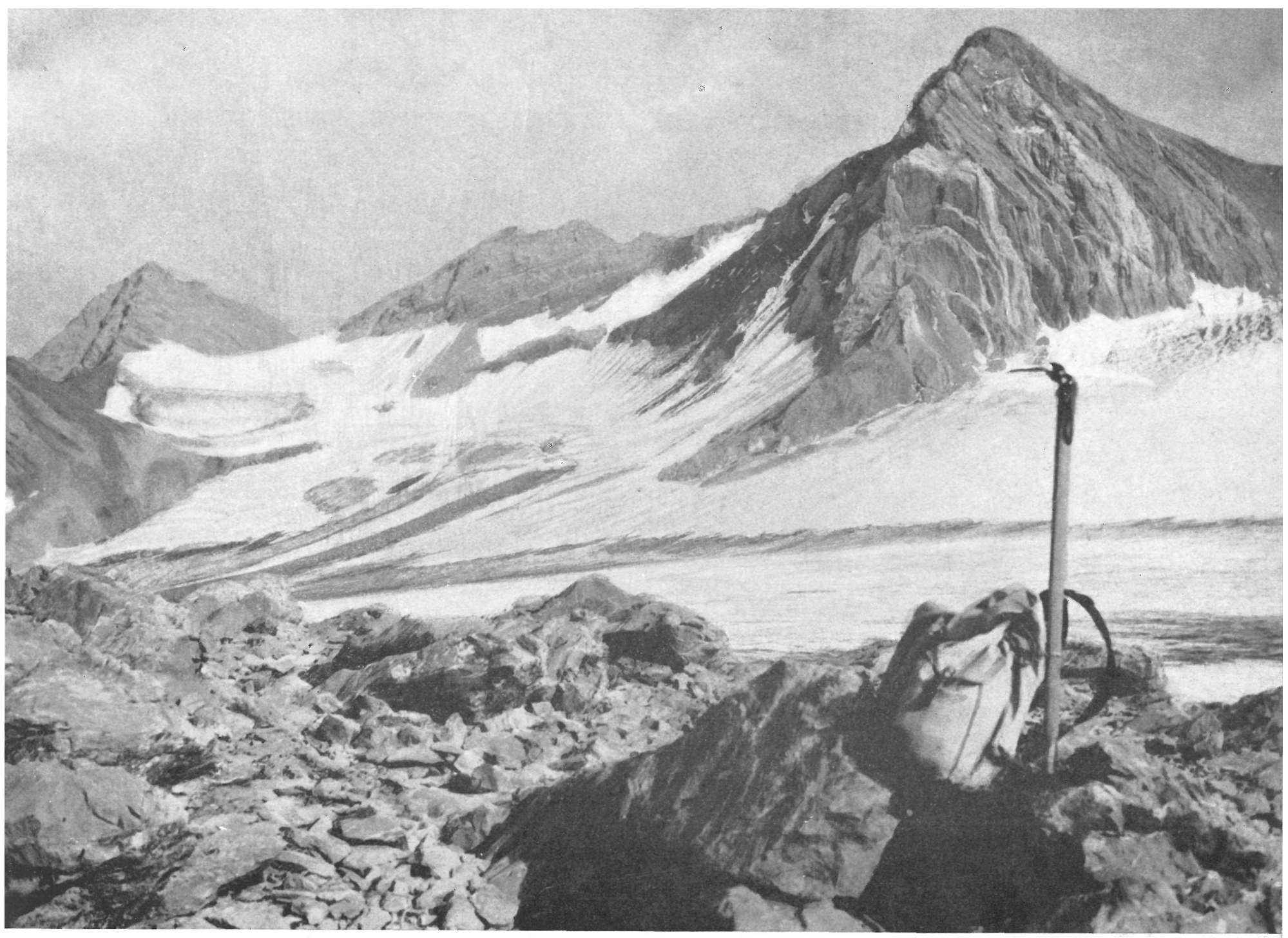

\title{
central de Vorarlberg
}

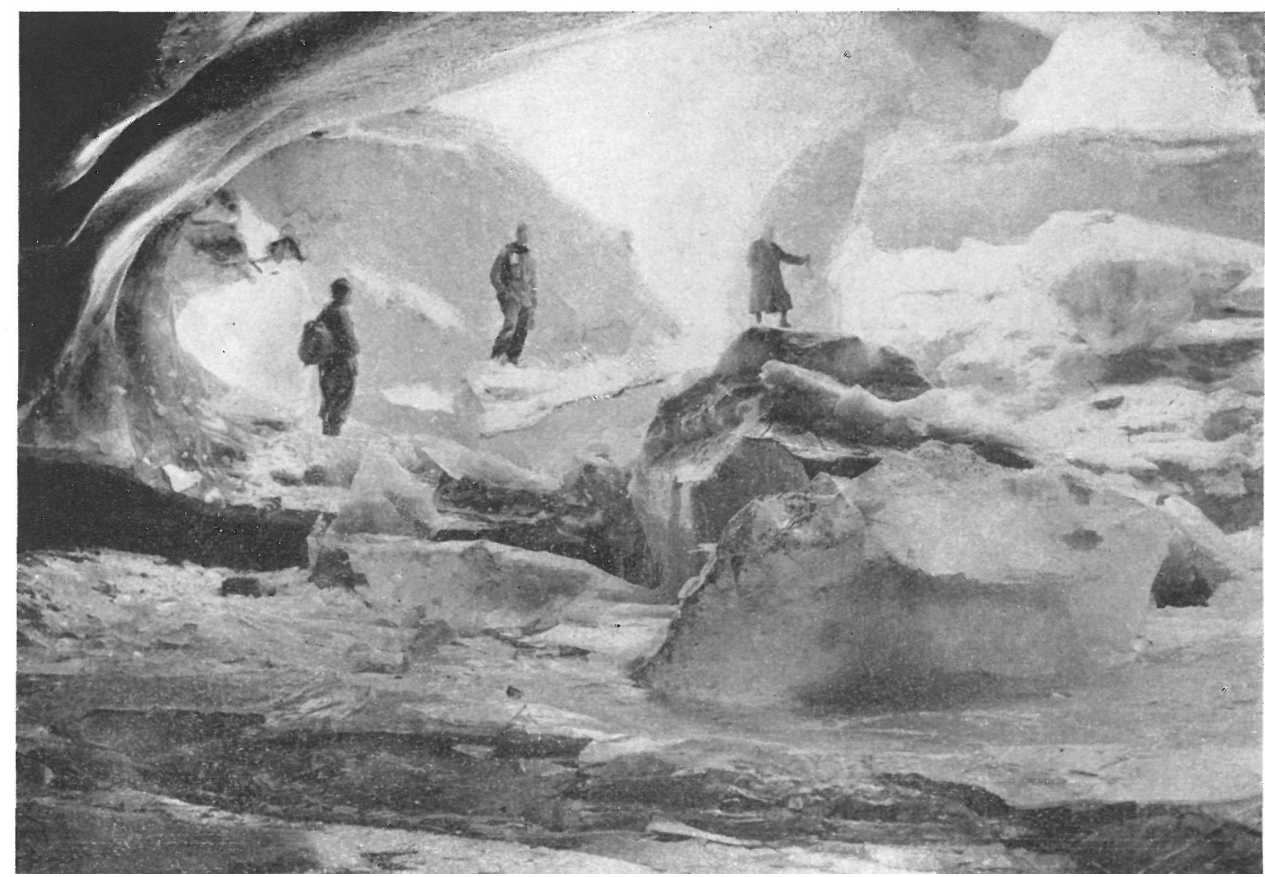

$531 \cdot 53$ 


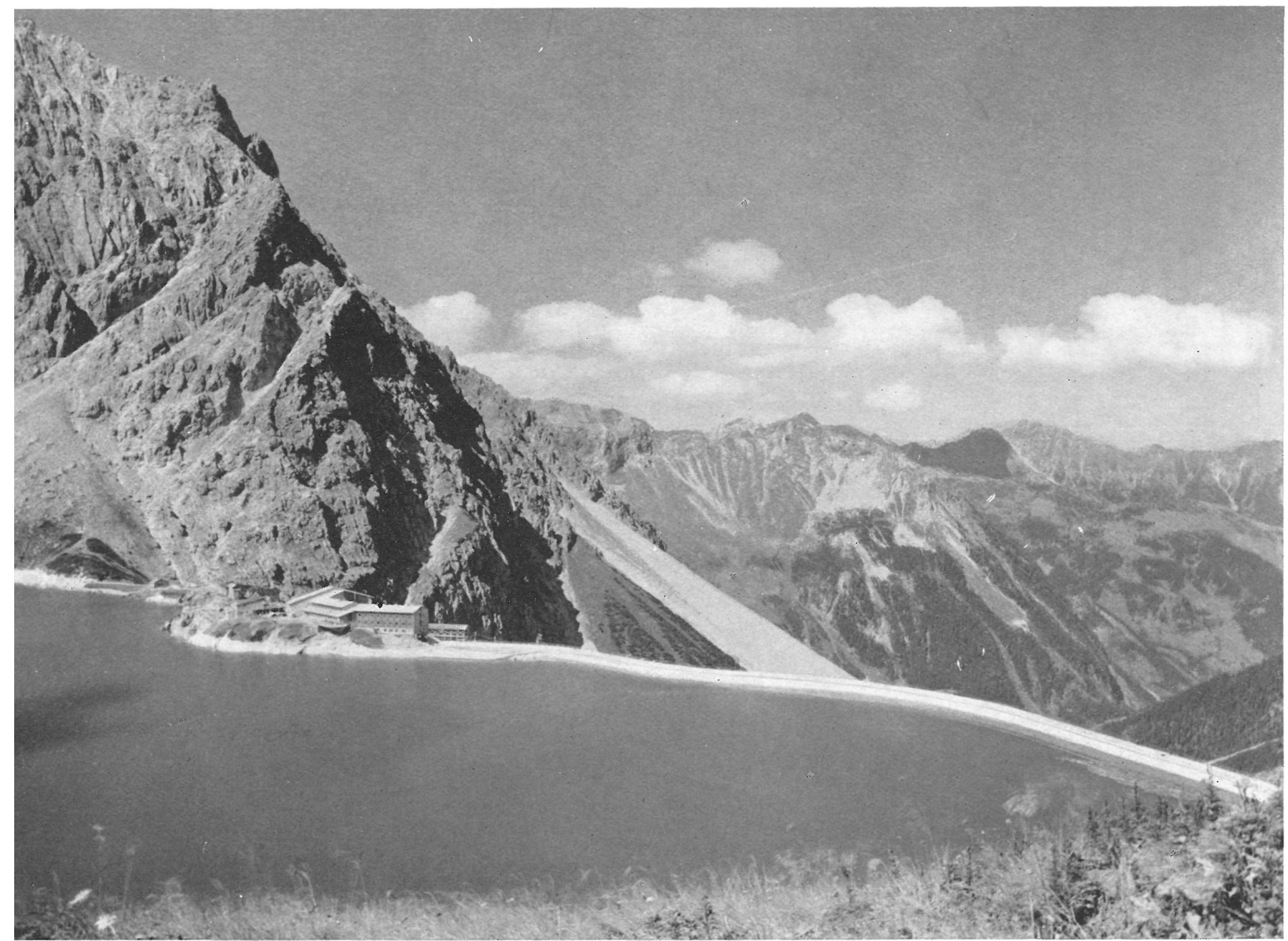

El desnivel aprovechable actualmente es de $1.000 \mathrm{~m}$ para la Central de Lünersee y de unos $350 \mathrm{~m}$ para la Central de Rołund. El lago natural de Lünersee se halla a $1.970 \mathrm{~m}$ de altitud.

La Central, a cielo abierto, de Lünersee, se halla en Latschau, y cuenta con un salto de $992,25 \mathrm{~m}$. Las aguas aprovechadas por esta Central se retienen en una cubeta.

El lago Lünersee tiene una capacidad de $76 \mathrm{hm}^{3}$, de los que recibe anualmente $17 \mathrm{hm}^{3}$ del glaciar Brandner.

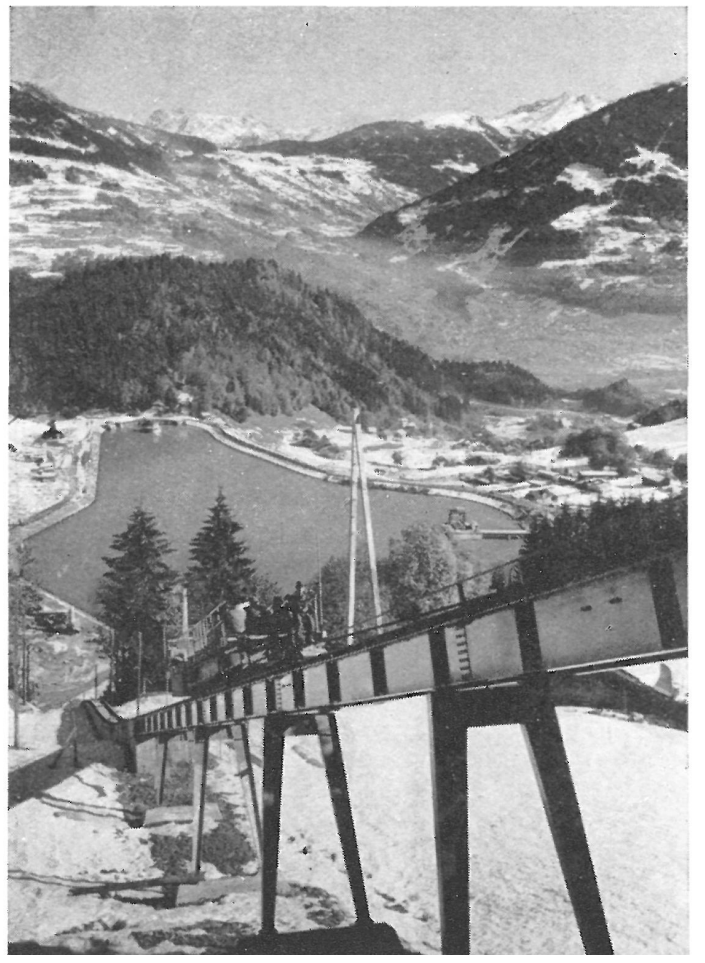




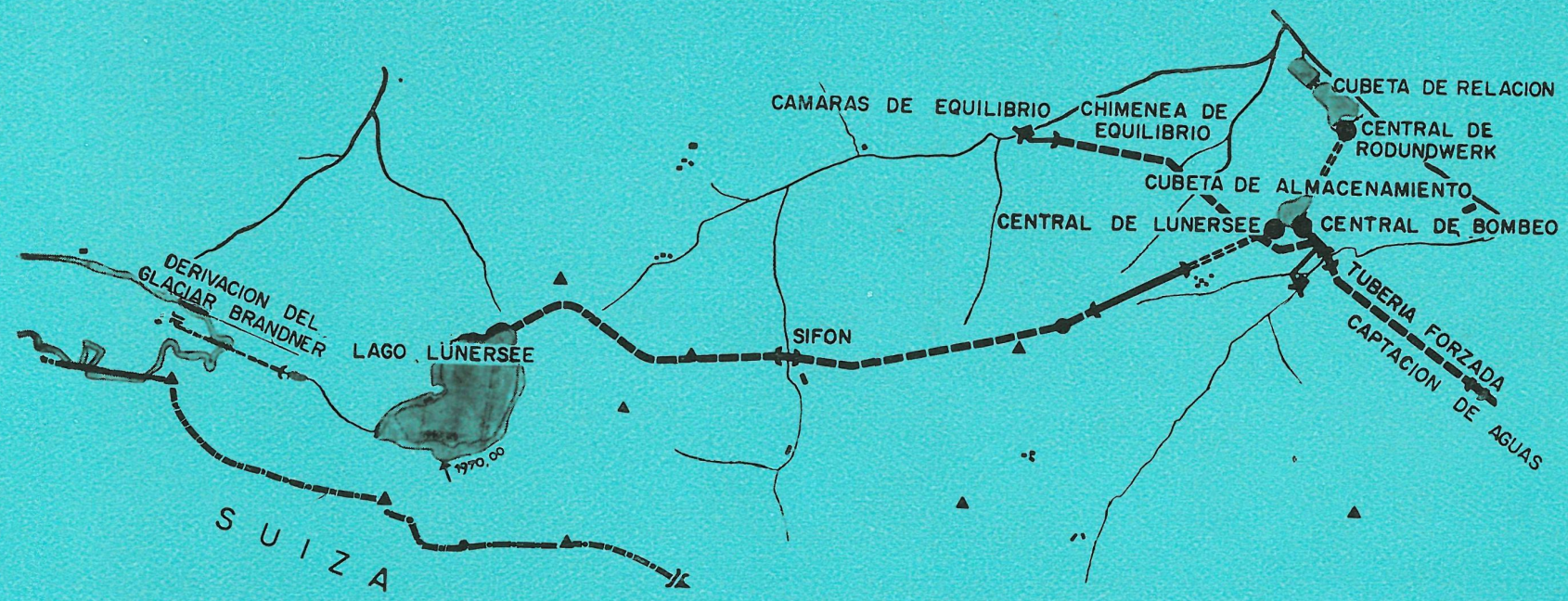

planta general

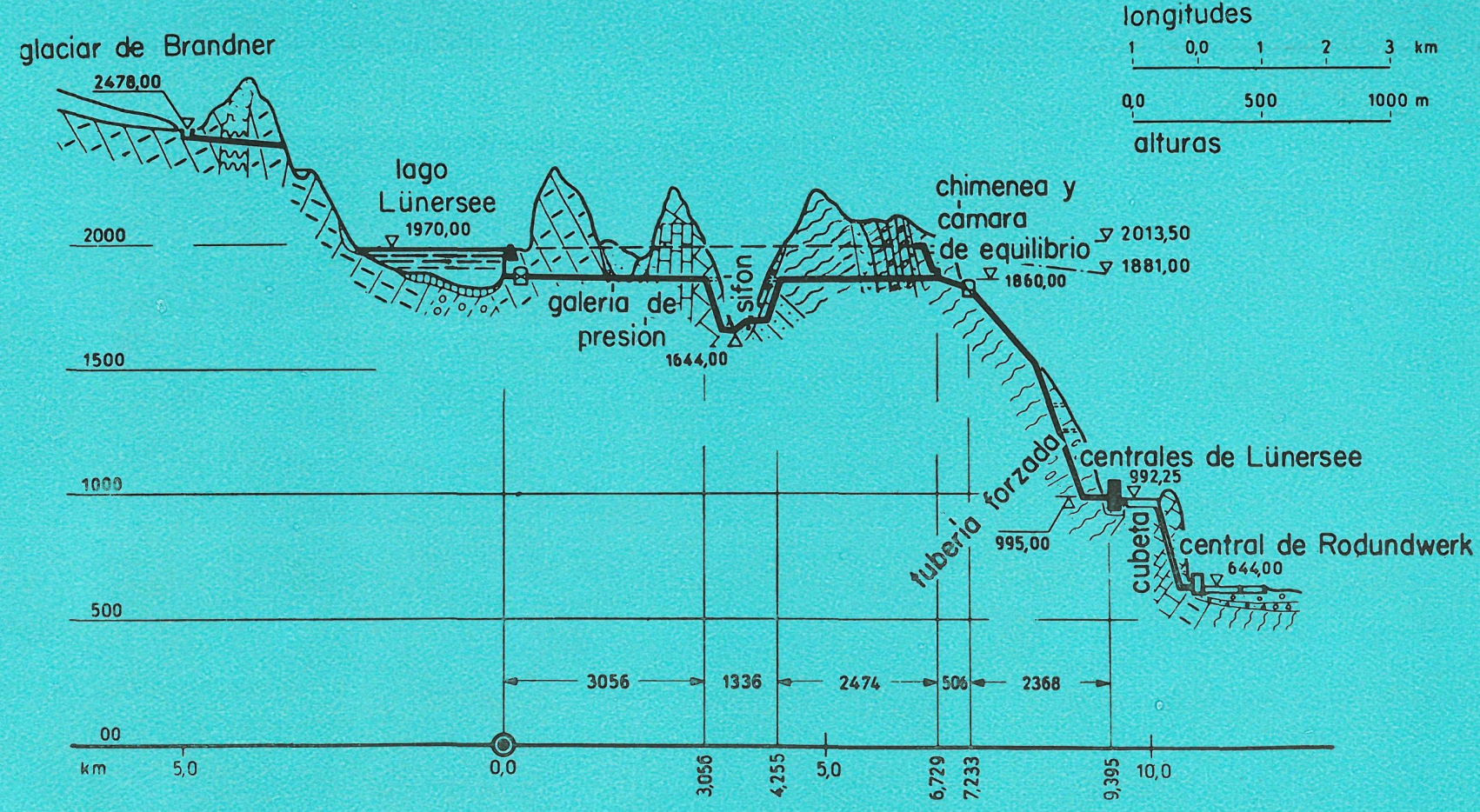

sección longitudinal 


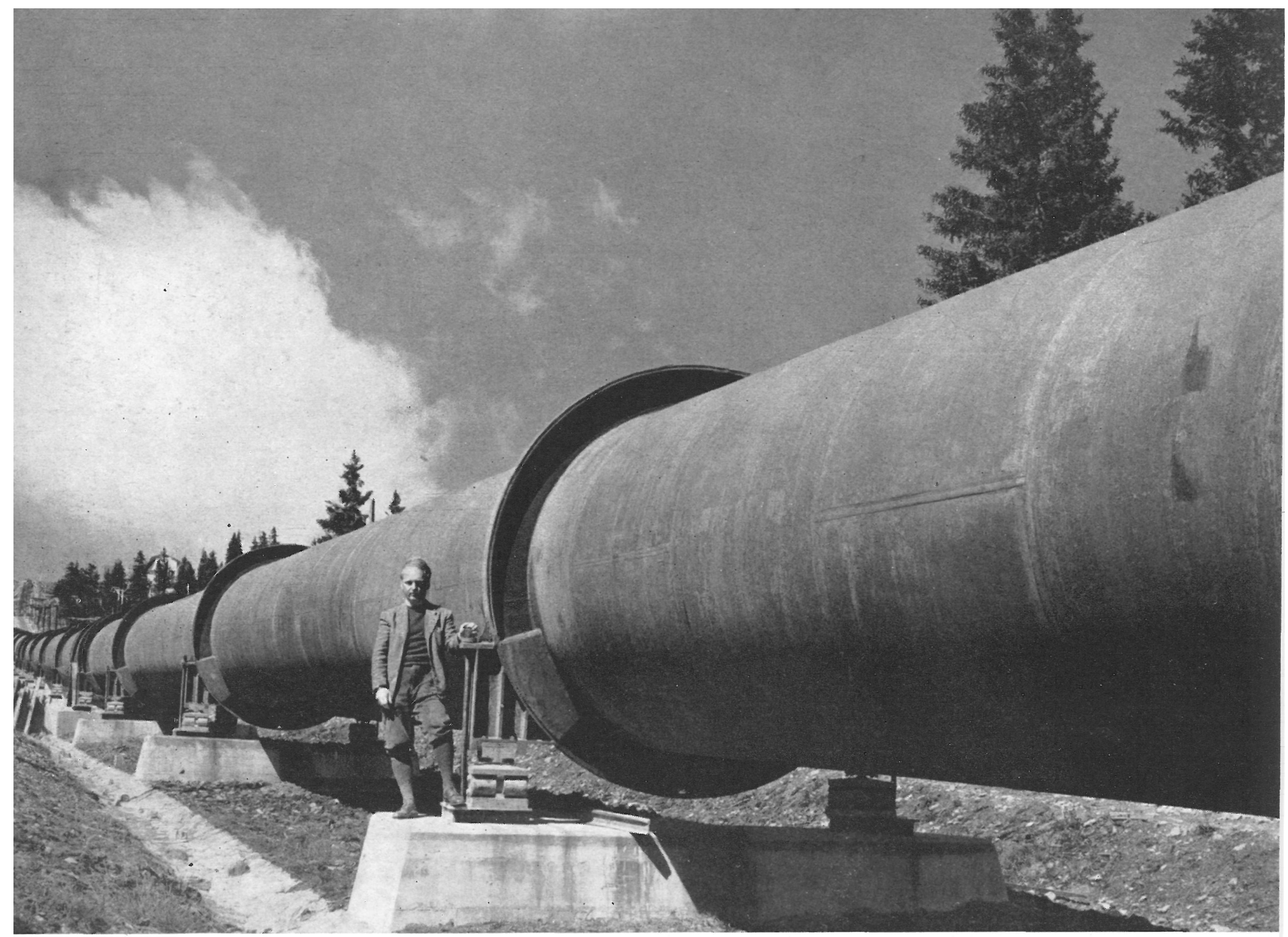

El resto son aguas aportadas por bombeo. El agua para el bombeo se acumula en la cubeta de aguas abajo de la Central, y procede de captaciones de arroyos próximos cuyo volumen se eleva por bombeo a la citada cubeta por medio de tres bombas. Las aguas del lago se recogen en el período de mayo a agosto.

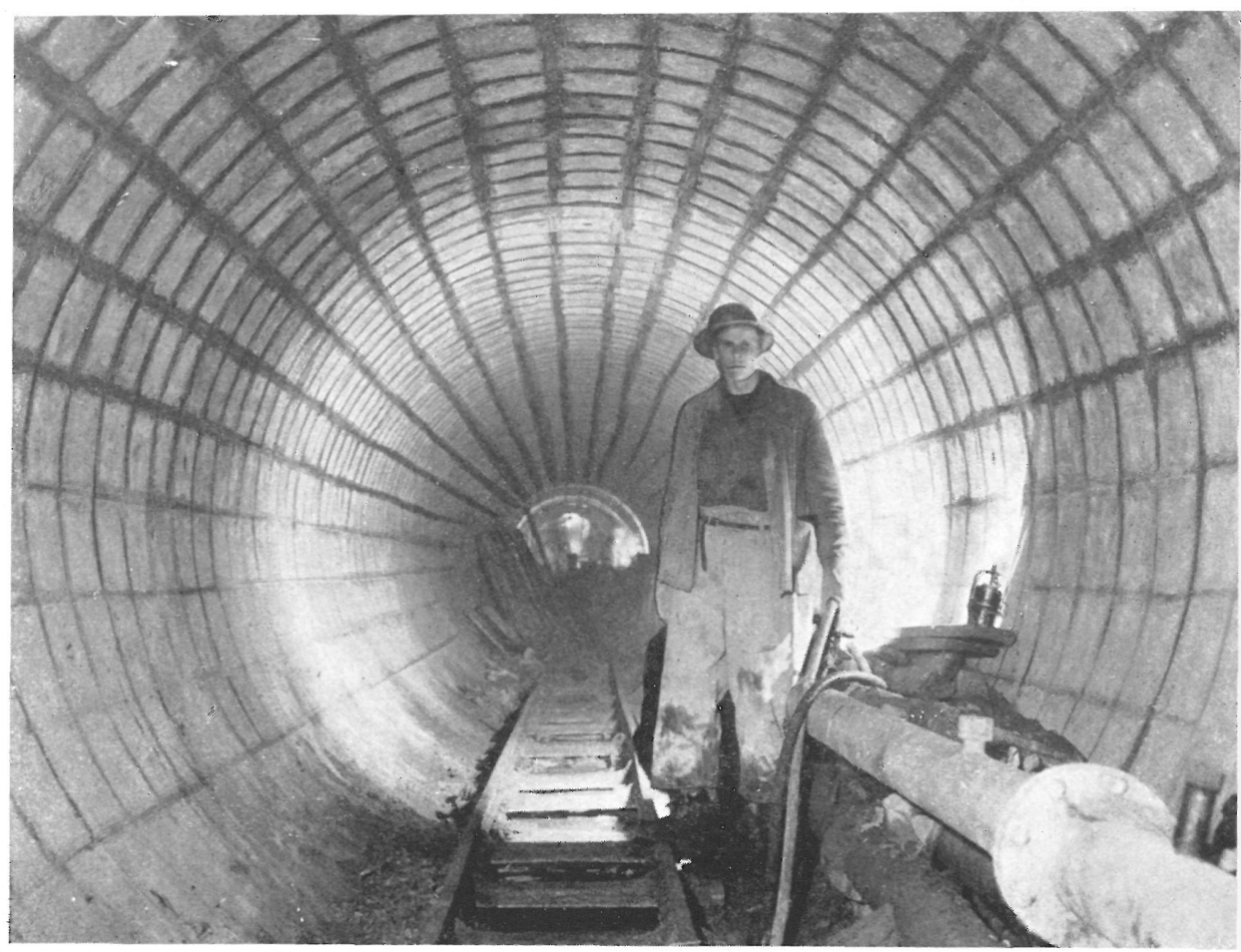




\section{Juego de energías}

En servicio normal anual, el bombeo requiere $198 \times 10^{6}$ $\mathrm{kWh}$, de las que son recuperables $88 \times 10^{6}$. Los $76 \mathrm{hm}^{3}$ de agua embalsada corresponden a una producción de $152 \times 10^{6}$ kWh de la Central Lünersee y de $57 \times 10^{6}$ de la Central de Rodund, es decir, $209 \times 10^{6} \mathrm{kWh}$ en total.

Aparte de esta explotación regular anual, la Central puede trabajar en servicio alterno adicional en cualquier período del año, lo que permite una producción adicional de $80 \times 10^{6} \mathrm{kWh}$ en verano y $121 \times 10^{6}$ en invierno. La energía necesaria que ha de aportarse para ello es de $135 \times 10^{6} \mathrm{kWh}$ en verano y de $203 \times 10^{6} \mathrm{kWh}$ en invierno 0 , lo que es lo mismo, $338^{\circ} \times 10^{6} \mathrm{kWh}$ en total.

La producción adicional combinada con la regular será de $80 \times 10^{6} \mathrm{kWh}$ en verano y 121 en invierno, que nos da un total de $201 \times 10^{6} \mathrm{kWh}$. La energía necesaria para eI bombeo en esta producción es de $135 \times 10^{6} \mathrm{kWh}$ en verano y de 203 en invierno, que arroja un total de $338 \times 10^{6} \mathrm{kWh}$ anuales. La producción total será, pues, de $353 \times 10^{6} \mathrm{kWh}$ de la Central de Lünersee y de 57 de la de Rodund, es decir, un total de $410 \times 10^{6} \mathrm{kWh}$, lo que exige una aportación de $536 \times 10^{6} \mathrm{kWh}$ para el bombeo.

\section{El salto}

El lago tiene una capacidad de $76 \mathrm{hm}^{3}$, se halla a $1.970 \mathrm{~m}$ de altitud, tiene $73 \mathrm{~m}$ de profundidad, y lo cierra una presa de gravedad de $41.000 \mathrm{~m}^{3}$ de hormigón, $28 \mathrm{~m}$ de altura, $380 \mathrm{~m}$ de longitud en coronación y dispone de un aliviadero de superficie, canal de descarga y desagüe de fondo. Dispone la presa de una toma de aguas, rejillas y la correspondiente compuerta o válvula de cierre.

Del glaciar Brandner parte una galería de captación de $6,1 \mathrm{~m}^{3}$ de capacidad y $1,34 \mathrm{~km}$ de longitud, a través de la cual discurre el agua libremente.

De la toma de la presa parte una galería de $3,06 \mathrm{~km}$ de longitud y de $3,5 \mathrm{~m}$ de diámetro, sometida a una presión de 12 atmósferas. A partir de este primer trozo se extiende otro, de $1,34 \mathrm{~km}$ de longitud, de 2,40 a $2,60 \mathrm{~m}$ de diámetro, blindada con una camisa metálica de 14 a $28 \mathrm{~mm}$, que salva un desnivel de $233 \mathrm{~m}$ y una presión correspondiente a un desnivel de $344 \mathrm{~m}$. De este trozo parte otro, de $2,47 \mathrm{~km}$ de longitud, $3,5 \mathrm{~m}$ de diámetro y que se halla sometido a una presión de 12 atmósferas.

La parte superior del salto, al final de estos trozos, cuenta con una válvula de paso y cierre, en cuya parte de aguas arriba parte la chimenea y cámaras de equilibrio con un desnivel total de $128 \mathrm{~m}$. La cámara inferior tiene una capacidad de $1.715 \mathrm{~m}^{3}$ y una longitud de $66 \mathrm{~m}$. La chimenea tiene $151 \mathrm{~m}$ de longitud y $38 \mathrm{~m}^{2}$ de sección horizontal. La cámara superior tiene una capacidad de $1.590 \mathrm{~m}^{3}$ y una longitud 
de $90 \mathrm{~m}$. La variación máxima superior al nivel medio es de $18 \mathrm{~m}$ y el descenso de nivel es de 28 metros.

La primer galería que constituye el último tramo tiene $500 \mathrm{~m}$ de longitud y $3,20 \mathrm{~m}$ de diámetro, y está provista de una camisa metálica de 15 a $19 \mathrm{~mm}$ de espesor. Al final de este tramo se encuentra el cierre de que antes hemos dado referencia. La segunda galería de este tramo, de $1.020 \mathrm{~m}$ de longitud, constituye la galería forzada, de $2,25 \mathrm{~m}$ de diámetro y un blindaje de 22 a $38 \mathrm{~mm}$ de espesor. La tercera galería o pozo, de $1,35 \mathrm{~km}$ de longitud y de 2,05 a $2,15 \mathrm{~m}$ de diámetro, tiene una camisa de 26 a $65 \mathrm{~mm}$ de espesor. Al final de este pozo se halla el canal de distribución, cuyas bifurcaciones están formadas por tubos de $67 \mathrm{~mm}$ de espesor de pared. El peso total de las tuberías de acero es de 8.000 toneladas.

La Central dispondrá de seis grupos verticales que trabajarán a $750 \mathrm{rpm}$. Cada grupo dispone de su generador, motor y turbina. La instalación lleva un regulador de presión hidráulico, dotado del correspondiente acoplamiento con la bomba.

Tiene una altura el salto de $977,75 \mathrm{~m}$ y el gasto hidráulico es de $31,5 \mathrm{~m}^{3} / \mathrm{s}$. La capacidad total de bombeo es de $26,3 \mathrm{~m}^{3} / \mathrm{s}$ (seis grupos trabajando a 50 períodos). La instalación tiene, además, un motor generador que trabaja a $56 \mathrm{kVA}$ y a $10,5 \mathrm{kV}$ que, con un salto útil de $972 \mathrm{~m}$, requiere un caudal de 5,52 metros cúbicos/segundo; también se dispone de un regulador hidráulico de presión de sincronización, de $32.000 \mathrm{HP}, \mathrm{y}$ una bomba de cinco fases, de $67.200 \mathrm{HP}$ de potencia, que trabaja con una impulsión de $897 \mathrm{~m}$ y una capacidad de $4,81 \mathrm{~m}^{3} / \mathrm{s}$ trabajando a 50 periodos. Dispone la instalación, además, de tres bombas que impelen de 14 a $24 \mathrm{~m}$ de altura con una capacidad de 4 a $8 \mathrm{~m}^{3} / \mathrm{s}$ trabajando a $260 \mathrm{rpm}$. A estas bombas le suministra la energía una turbina de 3.390 HP que se alimenta con una toma de tubería forzada.

E1 rendimiento medio de la instalación es de $217 \times 16^{6}$ $\mathrm{kWh}$ con las aguas naturales y de $253 \times 10^{6} \mathrm{kWh}$ con aguas de bombeo. Con cinco grupos el rendimiento es de $186 \times 10^{6}$ $\mathrm{kWh}$ trabajando con aguas recogidas y de $213 \times 10^{6} \mathrm{kWh}$ trabajando con las aguas procedentes del bombeo.

La potencia instalada es de $224.000 \mathrm{~kW}$, y la correspondiente al bombeo se eleva a $252.000 \mathrm{~kW}$ con 50 periodos.

La energía se transporta a la Central de Bürs por medio de una doble línea a $220 \mathrm{kV}$. Los cables de esta línea son de aluminio con alma de acero, la longitud es de $10,5 \mathrm{~km}$ y tiene 37 soportes.

La importancia energética de este aprovechamiento es de consideración. Todas las maniobras de mando se realizan por medio de pulsadores. El regulador hidráulico de sincronización hace posible un cambio rápido de la transformación de producción a bombeo y conexión con red. Con un rendimiento en turbinas de $217 \times 10^{6} \mathrm{kWh}$ y una producción con aguas de bombeo de $253 \times 10^{6} \mathrm{kWh}$ resulta, que una vez en servicio los seis grupos la producción se elevará a $470 \times 10^{6} \mathrm{kWh}$. 


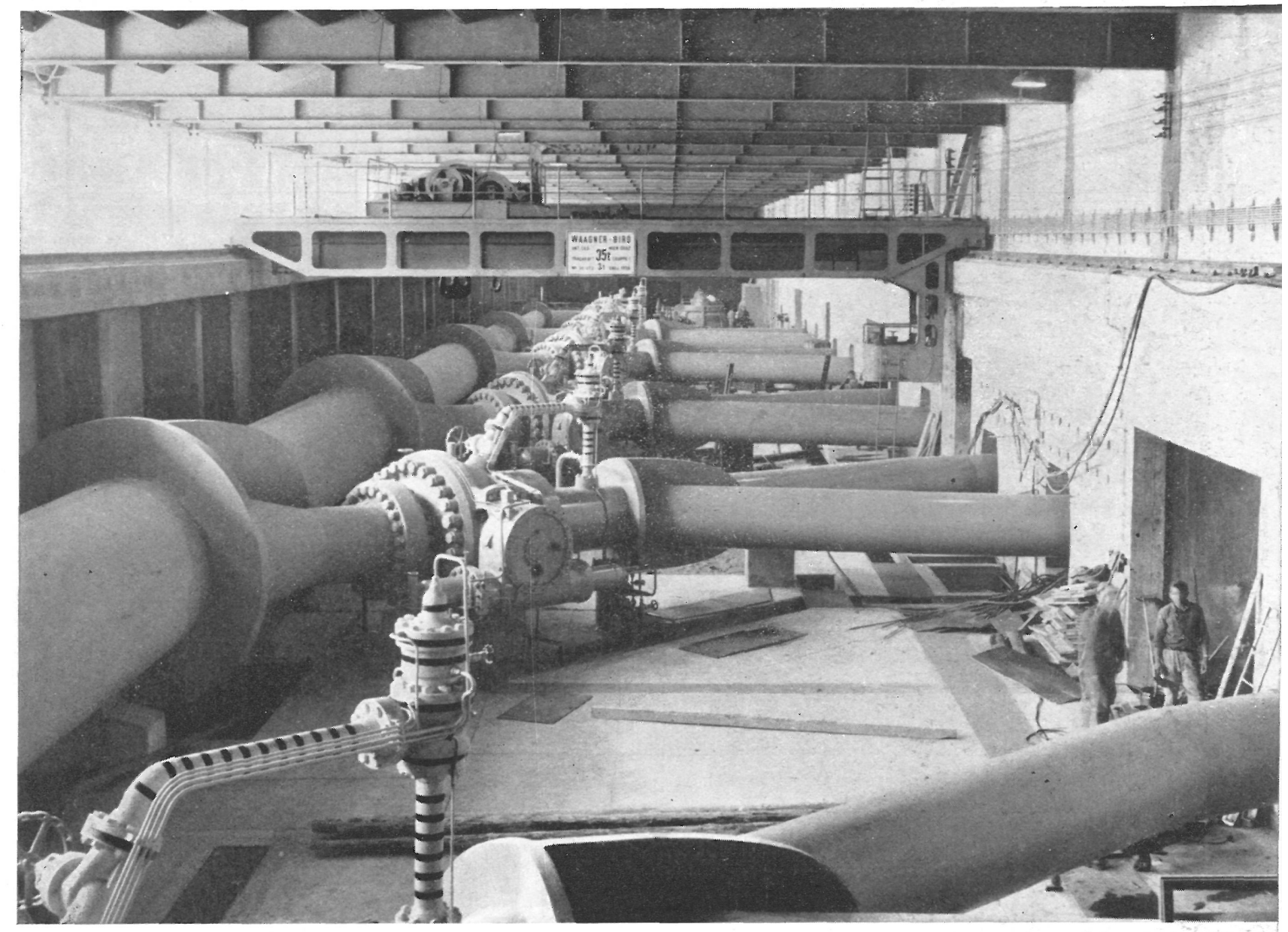

La Central Lünersee actúa sobre la red como un gran volante de inercia, absorbiendo energía de la red en los períodos muertos y alimentándola en los momentos de puntas, lo que permite una regulación de las oscilaciones de consumo, tanto diarias como anuales.

Este aprovechamiento, de puntas, aporta una mejora notable a la red general austríaca de distribución y, además, cabe la posibilidad de un ulterior aprovechamiento de $200 \mathrm{~m}$ más de caída o desnivel utilizable.

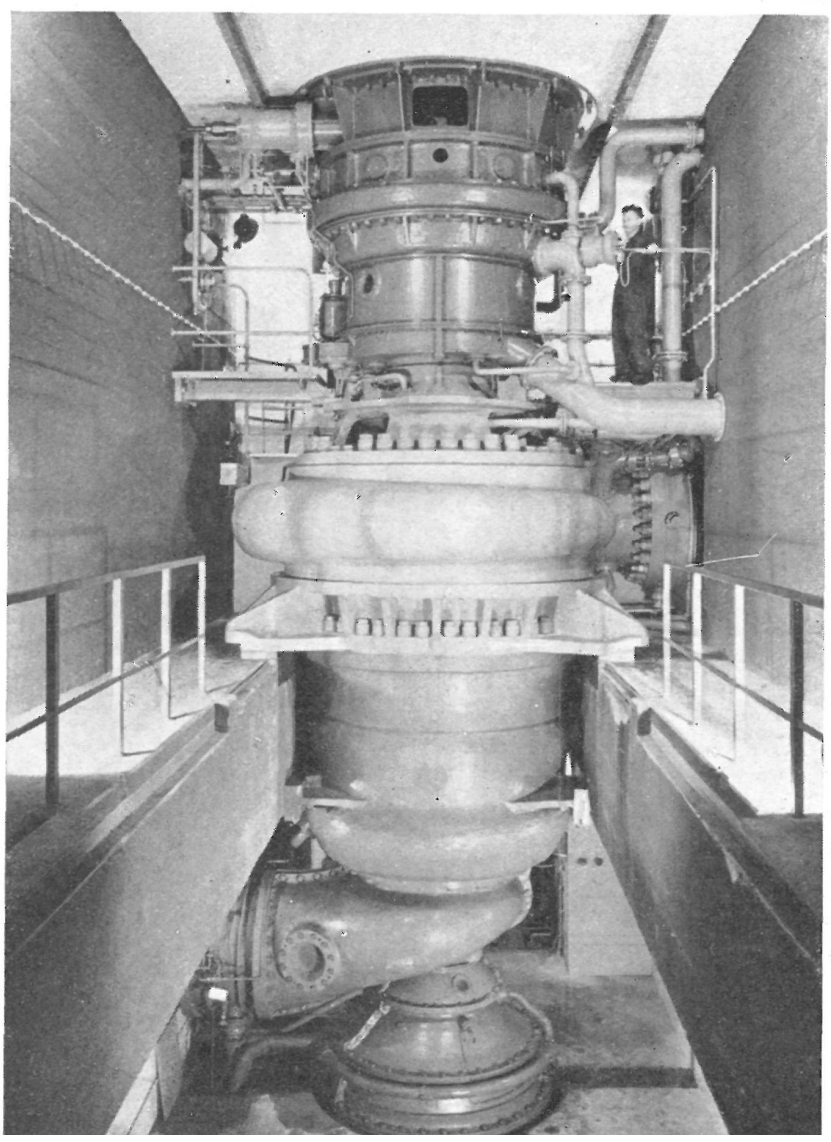



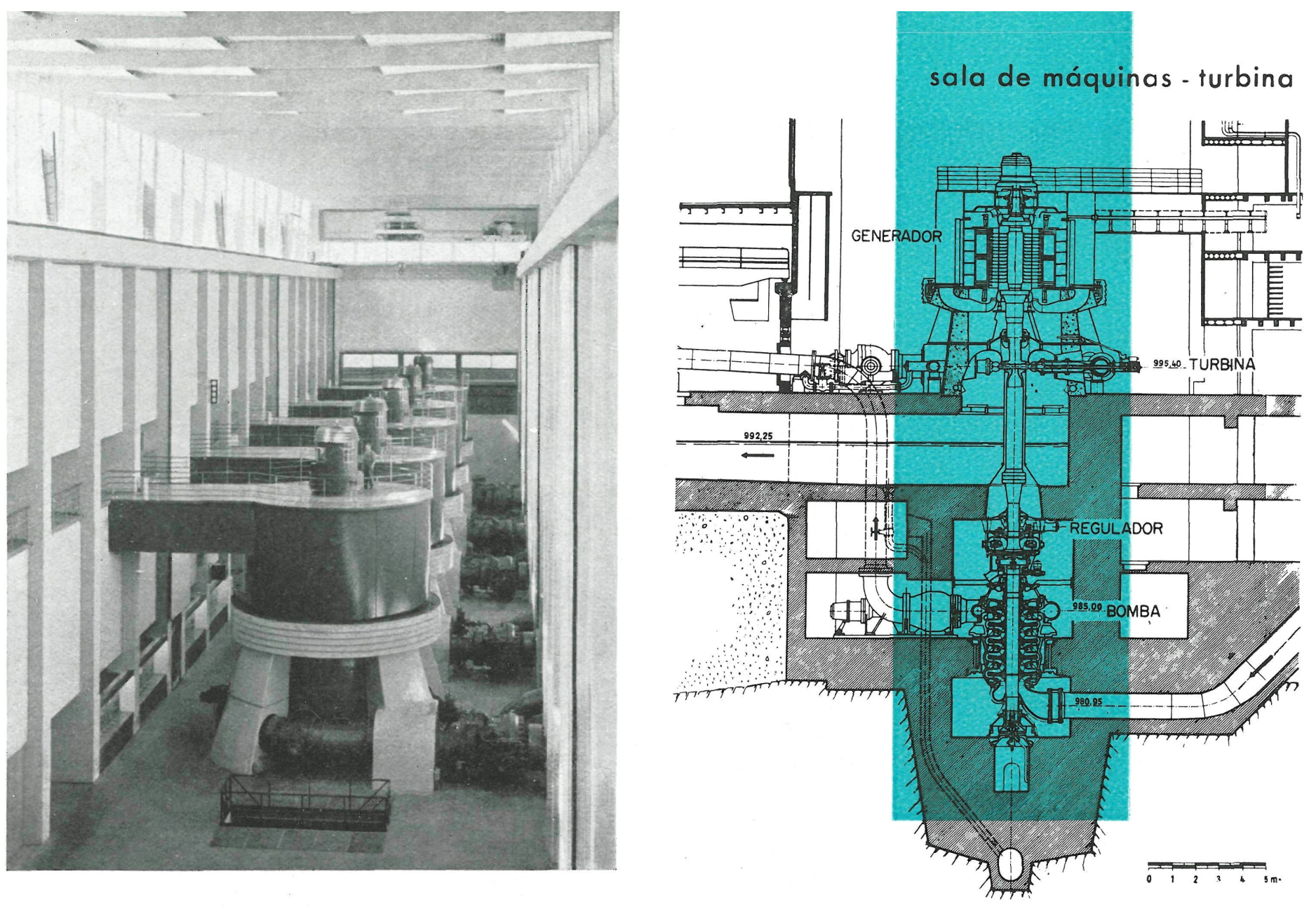

descarga de turbinas

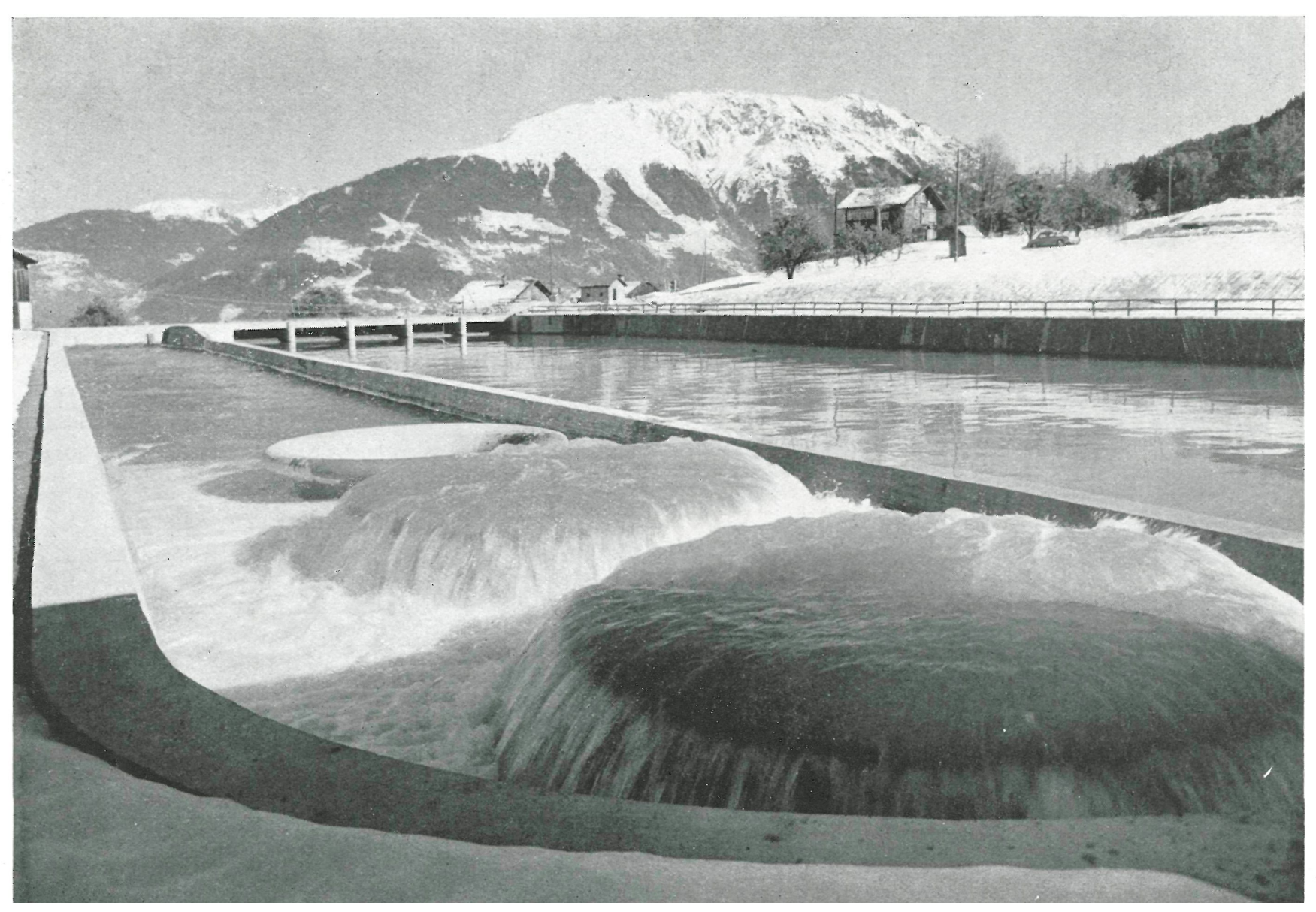

